\title{
Aggregating local image descriptors into compact codes
}

\author{
Hervé Jégou, Florent Perronnin, Matthijs Douze, Jorge Sánchez, Patrick Pérez, Cordelia Schmid
}

\begin{abstract}
This paper addresses the problem of large-scale image search. Three constraints have to be taken into account: search accuracy, efficiency, and memory usage. We first present and evaluate different ways of aggregating local image descriptors into a vector and show that the Fisher kernel achieves better performance than the reference bag-of-visual words approach for any given vector dimension. We then jointly optimize dimensionality reduction and indexing in order to obtain a precise vector comparison as well as a compact representation. The evaluation shows that the image representation can be reduced to a few dozen bytes while preserving high accuracy. Searching a 100 million image dataset takes about $250 \mathrm{~ms}$ on one processor core.
\end{abstract}

Index Terms - image search, image retrieval, indexing

\section{INTRODUCTION}

This paper proposes an image indexing scheme for very large databases. Image search consists here in finding in a large set of images the ones that represent the same object or location possibly viewed from different viewpoints and in the presence of occlusions and clutter. Typical applications include finding locations [1] and particular objects [2], as well as detecting partial image duplicates on the web [3] and deformed copies [4]. We do not consider specific visual recognition tasks, such as face detection and recognition, which require the use of specific descriptors and detectors. We also do not consider semantic queries such as "retrieve all images containing cars", which are usually addressed by supervised classification techniques, such as those evaluated in the PASCAL visual object recognition competition [5].

The bag-of-words (BOW) representation is the most widely adopted method for this purpose [6]. There are three reasons for the success of the BOW representation. First, this representation benefits from powerful local descriptors, such as the SIFT descriptor [7] or more recent ones [8], [9], [10]. Second, the BOW vector representation can be compared with standard distances. Third, the BOW vector can be high dimensional [2], [1], in which case the vectors are sparse and inverted lists can be used to implement efficient search [6]. However, two factors limit the number of images that can be indexed in practice: the search time itself, which becomes prohibitive when considering more than 10 million images, and the memory usage per image.

In this paper, we address the problem of searching for the most similar images to a given query in a very large image database (a hundred million images). We put an emphasis on the joint optimization of three constraints: the search

Preprint: Author version. accuracy, its efficiency and the memory usage. The last two are related [11], as search efficiency can be approximated by the amount of memory visited during the search. Most similar to our work is the approach of [12], which proposes an approximate nearest neighbor search for BOW features. However, this method is limited to small vocabularies and, thus, obtains a relatively low search accuracy. Moreover, an image needs to be encoded by more than a hundred bytes to approximate the search accuracy of the initial representation.

The efficiency problem was partially addressed by the min-Hash approach [13], [14] and the pre-filtering method of [15]. However, these techniques still require a significant amount of memory per image, and are more relevant in the context of near-duplicate image detection, as their search accuracy is significantly lower than BOW. Some authors address the efficiency and memory constraints by using GIST descriptors [16], and by converting them to compact binary vectors [17], [11], [18]. These approaches suffer from the limited robustness of the global GIST descriptor to geometric transformations [19], and none fulfills jointly the three aforementioned constraints: [11], [18] require an exhaustive search while [17] is memory consuming due to the redundancy of the LSH algorithm. In contrast, our approach obtains a higher accuracy with a very compact representation, i.e., codes of 20 bytes. This performance is achieved by optimizing:

1) the representation, i.e., how to aggregate local image descriptors into a vector representation;

2) the dimensionality reduction of these vectors;

3) the indexing algorithm.

These steps are closely dependent: a high-dimensional image representation usually provides better exhaustive search results than a low-dimensional one. However, high dimensional vectors are more difficult to index efficiently. A low dimensional vector can be indexed efficiently, but its discriminative power may be too low.

Our first contribution is an image representation of moderate dimensionality that provides excellent search accuracy. Our representation is based on Fisher kernels [20], a powerful tool to transform an incoming variable-size set of independent samples into a fixed-size vector representation. We assume that the samples are distributed according to a parametric generative model, in this case a Gaussian Mixture Model (GMM) estimated on a training set. Perronnin et al. [21] applied Fisher kernels in the context of image classification and large scale image search [22]. Experimental results demonstrate that this choice significantly outperforms BOW for the same size on most datasets. Moreover, it is cheaper to compute because 
fewer visual words are required. We also evaluate a simplified version of Fishers kernels, the Vector of Locally Aggregated Descriptors (VLAD) [23].

The description vectors are compared using the L2 distance. Their dimensionality is reduced to a few hundreds components by principal component analysis (PCA). To index the resulting vectors, we show the advantage of jointly optimizing the tradeoff between the dimensionality reduction and the indexing algorithm. We use the recent approximate nearest neighbor search method of [24], which performs similarity search with a technique derived from source coding, i.e., the encoded vectors can be approximately reconstructed. As a result, the error induced by PCA can be directly compared with the error resulting from the vector compression.

The paper is organized as follows. Section II introduces the datasets used to evaluate the components of our approach. The different strategies used to aggregate the descriptors, the BOW, the Fisher Kernel and the VLAD representations, are presented in Section III and compared in Section IV. We introduce the subsequent indexing stage in Section V, where a joint optimization of dimensionality reduction and indexing is proposed. Experimental results demonstrate the performance of our approach in section VI: the performance of BOW is obtained with an image representation of about 20 bytes. In terms of memory usage, search accuracy and efficiency, this is a significant improvement over the state of the art [12], as well as over our two prior conference publications [22], [23]. This paper is built upon these two prior publications and includes the following additions: (a) an analysis of the dimensionality reduction for local descriptors as well as for the final aggregated representation; (b) a theoretical and experimental comparison of the Fisher Kernel [21] and the VLAD representation [23], which allows improving our image search system; and (c) additional experiments, in particular on a dataset with 100 million images.

\section{DATASETS AND EVALUATION PROTOCOL}

To extract local features, we have used the experimental setup of [12] and the feature extraction software available on-line ${ }^{1}$. The regions of interest are extracted using the Hessian rotation- and affine-invariant region detector [25] and described by the SIFT descriptor [7]. We have used an independent image set for all the learning stages. The evaluation is performed on the datasets described below, and conducted by submitting each query image of the dataset to the evaluated system, which returns a list of images sorted by decreasing relevance.

The INRIA Holidays dataset [26] is a collection of 1491 holiday images, 500 of them being used as queries. The accuracy is measured by the mean Average Precision (mAP), as defined in [1].

The University of Kentucky Recognition Benchmark (UKB [2]) contains images of 2550 objects, each of which is represented by 4 images. The most common evaluation metric for this dataset counts the average number of relevant images

\footnotetext{
${ }^{1}$ http://lear.inrialpes.fr/people/jegou/data.php
}

(including the query itself) that are ranked in the first four positions when searching the 10200 images. This corresponds to 4 times the recall@4 measure, i.e., the best performance is 4.

The Oxford5K building dataset [1] contains 5062 photos gathered from Flickr with tags related to buildings in Oxford. Each of the 55 queries is defined by a rectangular region delimiting a building on an image. The correct results for a query are images of this building. The accuracy is measured by mAP.

The Flickr10M dataset allows to evaluate the performance of our method on a large scale with high quality pictures. It was obtained by downloading 10 million random images from Flickr. This image set is merged with the other datasets to evaluate the accuracy and efficiency on a large scale [12].

The Exalead100M dataset is used for large scale evaluation on up to $100 \mathrm{M}$ images and used in conjunction with the Copydays dataset described below. The images have been extracted from crawled web pages by Exalead, and were downscaled uniformly to 150 pixels in their larger dimension and compressed with a JPEG quality of 75 .

INRIA Copydays was designed to evaluate near-duplicate detection [19]. The dataset contains 157 original images. To obtain query images relevant in a copy detection scenario, each image of the dataset has been transformed with three different types of transformation:

- Image resizing (by a factor of 4 in dimension $=16$ in surface), followed by JPEG compression ranging from JPEG3 (very low quality) to JPEG75 (typical web quality). For each compression factor, 157 images are generated (one per database image).

- Cropping ranging from 5\% to $80 \%$ of the image surface. Here, we use only the queries with the cropping parameter fixed to $50 \%$ (one per database image, i.e., 157 query images in total).

- Strong transformations: print and scan, occlusion, change in contrast, perspective effect, blur, very strong cropping, etc. There is in total 229 transformed images, each of which has only a single matching image in the database.

The accuracy is measured by mAP. We merge the database with 10,000 distractor images from Flickr10M for most experiments. It is merged with images from Exalead100M for our very large scale evaluation. In this case, in order to obtain an image quality consistent with that of Exalead100M, the same down-scaling factor and JPEG compression is applied to the Copydays images.

For experiments on large image sets, we will use the recall@ $R$ metric, i.e., the rate of relevant images that are ranked in the top $R$ positions. This measure is relevant for two reasons. First, evaluating recall@ $R$ with respect to $R$ indicates the empirical distribution of the rank of the true positives. Second, if the rank $R$ is fixed to a small value (e.g., $R=100$ ), this indicates if a method provides a relevant shortlist for a subsequent precise (and more costly) direct image comparison based on geometric verification [1], [7]. 


\section{IMAGE VECTOR REPRESENTATION}

In this section, we introduce the different vector aggregation methods compared in this paper, namely the BOW representation, the Fisher Vector (FV) representation, and the VLAD vectors, which are shown to be an extremal case of FV. All these methods aim at aggregating $d$-dimensional local descriptors into a single vector representation.

\section{A. Bag-of-features}

The BOW representation groups together local descriptors. It requires the definition of a codebook of $K$ centroids ("visual words") usually obtained by k-means clustering. Each local descriptor of dimension $d$ from an image is assigned to the closest centroid. The BOW representation is the histogram of the number of image descriptors assigned to each visual word. Therefore, it produces a $K$-dimensional vector, which is subsequently normalized. There are several variations on how to normalize the histogram. When seen as an empirical distribution, the BOW vector is normalized using the Manhattan distance. Another common choice consists in using Euclidean normalization. The vector components are then weighted by idf (inverse document frequency) terms [2], [6]. In the following, we perform $L_{2}$ normalization of histograms and use the $i d f$ calculation of [6]. Several variations have been proposed to improve the quality of this representation. One among the most popular [27], [28] consists in using soft quantization techniques instead of a k-means.

\section{B. Fisher vector}

By counting the number of occurrences of visual words, BOW encodes the 0-order statistics of the distribution of descriptors. The Fisher vector extends the BOW by encoding high-order statistics (first and, optionally, second order). This description vector is the gradient of the sample's likelihood with respect to the parameters of this distribution, scaled by the inverse square root of the Fisher information matrix. As a result, it gives the direction, in parameter space, into which the learned distribution should be modified to better fit the observed data. In other terms, FV describes how the set of descriptors deviates from an average distribution of descriptors, modeled by a parametric generative model. It has been shown [20] that discriminative classifiers can be learned in this new representation space. In our image search framework, the FV is seen as a method to capture the information conveyed by a set of descriptors into a fixed-length signature.

The Fisher kernel framework. Let $X=\left\{x_{t}, t=1 \ldots T\right\}$ denote the set of $T$ local descriptors extracted from an image. We assume for now that the generation process of $X$ can be modeled by an image-independent probability density function $u_{\lambda}$ with parameters $\lambda^{2}$. Jaakkola and Haussler [20] proposed to describe $X$ by the vector :

$$
G_{\lambda}^{X}=\frac{1}{T} \nabla_{\lambda} \log u_{\lambda}(X) .
$$

\footnotetext{
${ }^{2}$ We abuse the notation to simplify the presentation: $\lambda$ denotes both the set of parameters of $u$ as well as the estimate of these parameters.
}

The gradient of the log-likelihood describes the contribution of the parameters to the generation process. Its dimensionality only depends on the number of parameters in $\lambda$. A natural kernel on these gradients is the "Fisher kernel" [20]:

$$
K(X, Y)=G_{\lambda}^{X} F_{\lambda}^{-1} G_{\lambda}^{Y}
$$

where $F_{\lambda}$ is the Fisher information matrix of $u_{\lambda}$ :

$$
F_{\lambda}=E_{x \sim u_{\lambda}}\left[\nabla_{\lambda} \log u_{\lambda}(x) \nabla_{\lambda} \log u_{\lambda}(x)^{\top}\right] .
$$

As $F_{\lambda}^{-1}$ is symmetric and positive definite, it has a Cholesky decomposition $F_{\lambda}^{-1}=L_{\lambda}^{\top} L_{\lambda}$. Therefore the kernel $K(X, Y)$ is advantageously rewritten as a dot-product between normalized vectors $\mathcal{G}_{\lambda}$, obtained as

$$
\mathcal{G}_{\lambda}^{X}=L_{\lambda} G_{\lambda}^{X} .
$$

We refer to $\mathcal{G}_{\lambda}^{X}$ as the Fisher vector $(\mathrm{FV})$ of $X$.

The FV image representation. We follow Perronnin and Dance [21] and choose $u_{\lambda}$ to be a GMM: $u_{\lambda}(x)=$ $\sum_{i=1}^{K} w_{i} u_{i}(x)$. We denote $\lambda=\left\{w_{i}, \mu_{i}, \sigma_{i}, i=1 \ldots K\right\}$ where $w_{i}, \mu_{i}$ and $\sigma_{i}$ are respectively the mixture weight, mean vector and variance matrix (assumed diagonal) of Gaussian $u_{i}$. The model $u_{\lambda}$ can be understood as a probabilistic visual vocabulary. It is trained offline on a large number of images using Maximum Likelihood (ML) estimation. In this work, we focus on the gradient with respect to the mean. We use the diagonal closed-form approximation of the Fisher information matrix of [21], in which case the normalization of the gradient by $L_{\lambda}=F_{\lambda}^{-1 / 2}$ is simply a whitening of the dimensions. Let $\gamma_{t}(i)$ be the soft assignment of descriptor $x_{t}$ to the $i^{\text {th }}$ Gaussian:

$$
\gamma_{t}(i)=\frac{w_{i} u_{i}\left(x_{t}\right)}{\sum_{j=1}^{K} w_{j} u_{j}\left(x_{t}\right)} .
$$

Let $\mathcal{G}_{i}^{X}$ be the $d$-dimensional gradient with respect to the mean $\mu_{i}$ of Gaussian $i$. Assuming that the $x_{t}$ 's were generated independently by $u_{\lambda}$, we obtain after standard mathematical derivations:

$$
\mathcal{G}_{i}^{X}=\frac{1}{T \sqrt{w_{i}}} \sum_{t=1}^{T} \gamma_{t}(i) \sigma_{i}^{-1}\left(x_{t}-\mu_{i}\right) .
$$

The final vector $\mathcal{G}_{\lambda}^{X}$ is the concatenation of the $\mathcal{G}_{i}^{X}$ vectors for $i=1 \ldots K$ and is therefore $K d$-dimensional ${ }^{3}$. In comparison with the BOW, $d$ times fewer visual words are required to obtain a signature of the same length. Experimental results show that excellent results can be obtained even with a relatively small number of visual words $K$ : we typically consider values ranging from $K=16$ to $K=256$.

FV Normalization. The FV undergoes two normalization steps. First, a power normalization is performed by applying the following operator independently on each component:

$$
f(z)=\operatorname{sign}(z)|z|^{\alpha}
$$

${ }^{3}$ Taking the gradient with respect to the mean and variance leads to vectors of size $2 K d$. We can obtain vectors of the same size by taking the gradient with respect to the mean only on a GMM with $2 K$ Gaussians. In a set of preliminary experiments, we observed that for a fixed FV size the two options led to very similar results. 
with $0 \leq \alpha \leq 1$. We empirically observe that this step consistently improves the quality of the representation. Several complementary interpretations justify this transform. First, it reduces the influence of bursty visual elements, which were shown to corrupt the image similarity in [29]. Second, assuming the compound Poisson distribution as a good generative model of FVs, the power normalization can be interpreted as a variance stabilizing transform, which corrects the dependence between the variance and the mean. We provide an analysis of this interpretation in the Appendix provided as supplemental material. Variance stabilizing transforms have previously been applied to BOW histograms, e.g. in [30] for GMM modeling and in [31], [32] for better separability of linear classifiers.

We found experimentally that the optimal value for $\alpha$ depends on the number of Gaussians $K$. However, setting $\alpha=0.5$ consistently leads to near-optimal results on the range of $K$ values we consider.

The power normalized FV is then L2-normalized, which amounts to using the cosine similarity between FVs. It guarantees that if we query a database with one of its images, the first result will be the image itself if the inner product is used as a similarity measure - a desirable property.

Properties of the FV. We now assume that the descriptors $X=\left\{x_{t}, t=1 \ldots T\right\}$ of a given image are iid and follow a distribution $p$ which departs from the generic distribution $u_{\lambda}$. According to the law of large numbers (convergence of the sample average to the expected value when $T$ increases), we have

$$
\begin{aligned}
G_{\lambda}^{X} & =\frac{1}{T} \sum_{t=1}^{T} \nabla_{\lambda} \log u_{\lambda}\left(x_{t}\right) \\
& \approx \nabla_{\lambda} E_{x \sim p} \log u_{\lambda}(x) \\
& =\nabla_{\lambda} \int_{x} p(x) \log u_{\lambda}(x) d x .
\end{aligned}
$$

Now let us assume that we can decompose $p$ into a mixture of two parts: a background image-independent part which follows $u_{\lambda}$ and an image-specific part which follows an image-specific distribution $q$. Let $0 \leq \omega \leq 1$ be the proportion of imagespecific information contained in the image:

$$
p(x)=\omega q(x)+(1-\omega) u_{\lambda}(x) .
$$

We can rewrite:

$$
\begin{aligned}
G_{\lambda}^{X} & \approx \omega \nabla_{\lambda} \int_{x} q(x) \log u_{\lambda}(x) d x \\
& +(1-\omega) \nabla_{\lambda} \int_{x} u_{\lambda}(x) \log u_{\lambda}(x) d x .
\end{aligned}
$$

If the values of the parameters $\lambda$ were estimated with a ML process - i.e., to maximize (at least locally and approximately) $E_{x \sim u_{\lambda}} \log u_{\lambda}(x)$ - then:

$$
\nabla_{\lambda} \int_{x} u_{\lambda}(x) \log u_{\lambda}(x) d x=\nabla_{\lambda} E_{x \sim u_{\lambda}} \log u_{\lambda}(x) \approx 0 .
$$

Consequently, we have:

$$
G_{\lambda}^{X} \approx \omega \nabla_{\lambda} \int_{x} q(x) \log u_{\lambda}(x) d x=\omega \nabla_{\lambda} E_{x \sim q} \log u_{\lambda}(x) .
$$

This shows that the image-independent information is approximately discarded from the FV signature, and that an image is described by what makes it different from other images (on average). Such a decomposition of images into background and image-specific information has also been employed in BOW approach by Zhang et al. [33]. However, while the decomposition is explicit in [33], it is implicit in the FV case.

Relationship with BOW. We introduce

$$
\begin{aligned}
w_{i}^{X} & =\frac{1}{T} \sum_{t=1}^{T} \gamma_{t}(i), \\
\mu_{i}^{X} & =\frac{\sum_{t=1}^{T} \gamma_{t}(i) x_{t}}{\sum_{t=1}^{T} \gamma_{t}(i)} .
\end{aligned}
$$

where $w_{i}^{X}$ is the proportion of descriptors of $X$ soft-assigned to visual word $i$, i.e., this is the soft-BOW representation. The vector $\mu_{i}^{X}$ is the average of the descriptors of $X$ weighted by their probability of being assigned to Gaussian $i$ (i.e., loosely speaking, the average of the descriptors of $X$ assigned to Gaussian $i$ ). We can rewrite the gradient (6) as follows:

$$
\mathcal{G}_{i}^{X}=\frac{w_{i}^{X}}{\sqrt{w_{i}}} \sigma_{i}^{-1}\left(\mu_{i}^{X}-\mu_{i}\right) .
$$

This clearly shows that the FV extends the BOW representation. Not only does the FV count the number of descriptors assigned to each region in the space, it also encodes the approximate location of the descriptors in each region (relatively to the mean $\mu_{i}$ and the variance $\sigma_{i}$ ). The division by $\sqrt{w_{i}}$ in (17) can be interpreted as a BOW idf term: the descriptors which occur frequently are automatically discounted.

\section{VLAD: non probabilistic Fisher Kernel}

In [23], Jégou et al. proposed the VLAD representation. As for the BOW, a codebook $\left\{\mu_{1}, \ldots \mu_{K}\right\}$ is first learned using k-means. Each local descriptor $x_{t}$ is associated to its nearest visual word $\mathrm{NN}\left(x_{t}\right)$ in the codebook. For each codeword $\mu_{i}$, the differences $x_{t}-\mu_{i}$ of the vectors $x_{t}$ assigned to $\mu_{i}$ are accumulated:

$$
v_{i}=\sum_{x_{t}: \mathrm{NN}\left(x_{t}\right)=i} x_{t}-\mu_{i}
$$

The VLAD is the concatenation of the $d$-dimensional vectors $v_{i}$ and is therefore $K d$ dimensional. Algorithm 1 sums up the resulting algorithm. As for FV, the VLAD can then be power- and L2-normalized.

We now show that the VLAD is a simplified nonprobabilistic version of the FV: the VLAD is to the FV what $\mathrm{k}$-means is to GMM clustering. The k-means clustering can be viewed as a non-probabilistic limit case of GMM clustering when:

a) the mixture weights are equal, i.e., $w_{i}=1 / K, \forall i$,

b) covariance matrices are isotropic, i.e., $\sigma_{i}=\epsilon \mathbb{I}$ with $\epsilon \geq 0$,

c) the value $\epsilon$ converges to zero.

If we enforce hypotheses a) and b), Equation 6 simplifies to:

$$
\mathcal{G}_{i}^{X} \propto \sum_{t=1}^{T} \gamma_{t}(i)\left(x_{t}-\mu_{i}\right)
$$




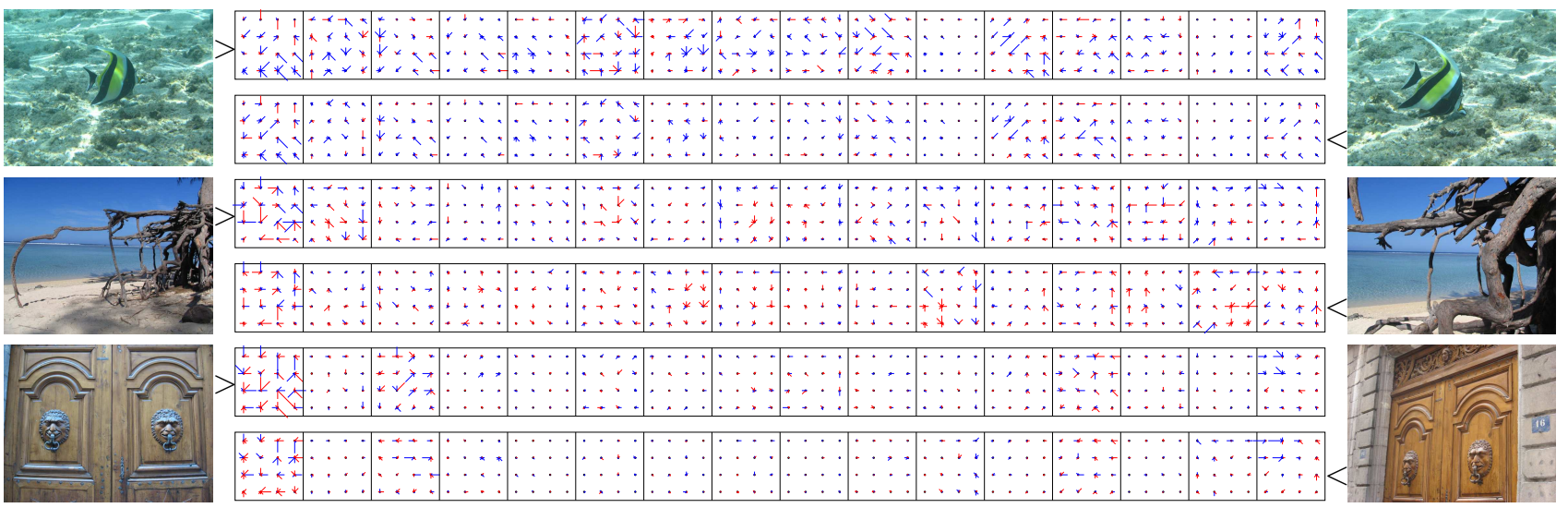

Fig. 1. Images and corresponding VLAD descriptors, for $K=16$ centroids. The components of the descriptor are represented like SIFT, with negative components in red.

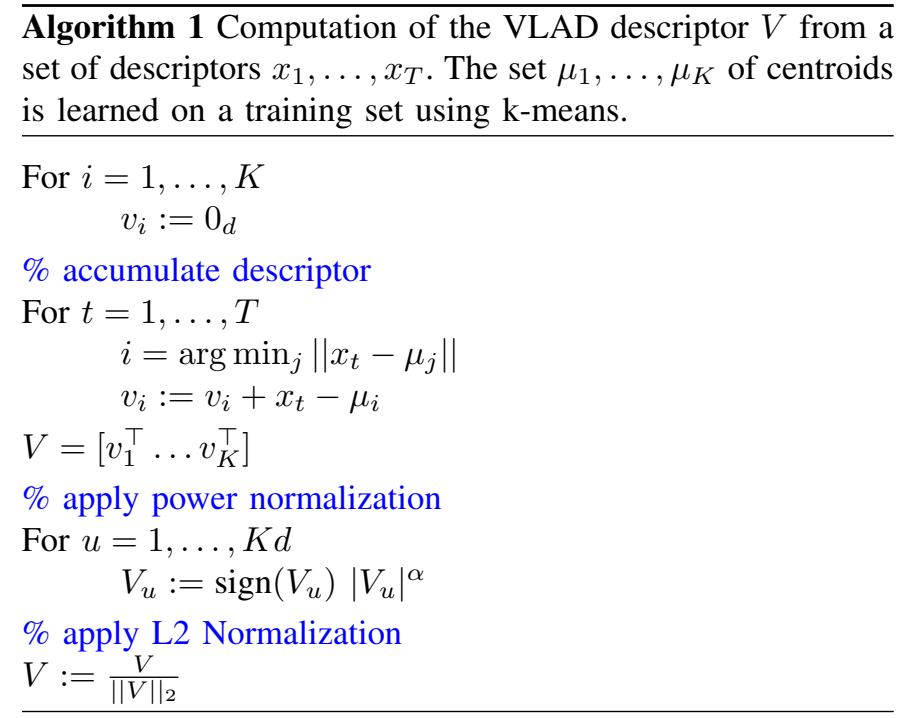

up to a factor independent of $\epsilon$ after normalization.

As $\epsilon \rightarrow 0$, the Gaussians converge to Dirac distributions and hypothesis c) makes the assignments $\gamma_{t}(i)$ binary: $\gamma_{t}(i)=1$ if $\mathrm{NN}\left(x_{t}\right)=i$ and $\gamma_{t}(i)=0$ otherwise. Therefore, as $\epsilon \rightarrow 0$ Equation 19 becomes

$$
\mathcal{G}_{i}^{X} \propto \sum_{x_{t}: \mathrm{NN}\left(x_{t}\right)=i} x_{t}-\mu_{i},
$$

where we recognize the VLAD representation.

Figure 1 depicts the VLAD representations associated with a few images, when aggregating 128-dimensional SIFT descriptors. The components of our descriptor map to components of SIFT descriptors. Therefore we adopt the usual $4 \times 4$ spatial grid with oriented gradients for each $v_{i}, i=1 \ldots K$, with $K=16$. In contrast to SIFT descriptors, a component may be positive or negative, due to the subtraction in Equation 18.

One can observe that the descriptors are relatively sparse (few values have a significant energy) and very structured: most high descriptor values are located in the same cluster, and the geometrical structure of SIFT descriptors is observable. Intuitively and as shown later, a principal component analysis is likely to capture this structure. For resembling images, the similarity of the descriptors is obvious.

\section{Dimensionality reduction on local descriptors}

Principal component analysis (PCA) is a standard tool [34] for dimensionality reduction: the eigenvectors associated with the most energetic eigenvalues of the empirical vector covariance matrix are used to define a matrix $M$ mapping a vector $x \in \mathbb{R}^{128}$ to a vector $x^{\prime}=M x$ in a lower-dimensional space.

We will show in next section that applying the Fisher Kernel framework directly on local descriptors leads to suboptimal results. Therefore, we apply a PCA on the SIFT descriptors to reduce them from 128 to $d=64$ dimensions. Two reasons may explain the positive impact of this PCA:

1. Decorrelated data can be fitted more accurately by a GMM with diagonal covariance matrices;

2. The GMM estimation is noisy for the less energetic components.

To confirm that these hypotheses are reasonable, we have performed two experiments on descriptor transformations. First, we applied a random rotation matrix after the PCA, that cancels its decorrelating effect. This significantly degrades the search quality. The second transformation is the PCA rotation without dimensionality reduction. This also degrades the retrieval performance, which means that the least energetic components are detrimental.

\section{EVALUATION OF THE AGGREGATION METHODS}

In this section, we evaluate and compare the different local aggregation methods described in Section III, BOW, FV, and VLAD, as well as the global GIST descriptor [16]. We analyze the impact of the number $K$ of centroids/mixture components and study the impact of dimensionality reduction at two stages of the algorithms:

- the local SIFT descriptors are reduced from 128 to 64 components using PCA (see Section III-D);

- the final VLAD, Fisher and BOW vectors are reduced from $D=K d$ to $D^{\prime}$ components using PCA.

In both cases, the PCA rotation matrices are learned on an independent image dataset. Note that in this section the evaluation is performed without the subsequent indexing stage.

Dimensionality reduction of local descriptors. Figure 2 compares the FV and VLAD representations on the Holidays 


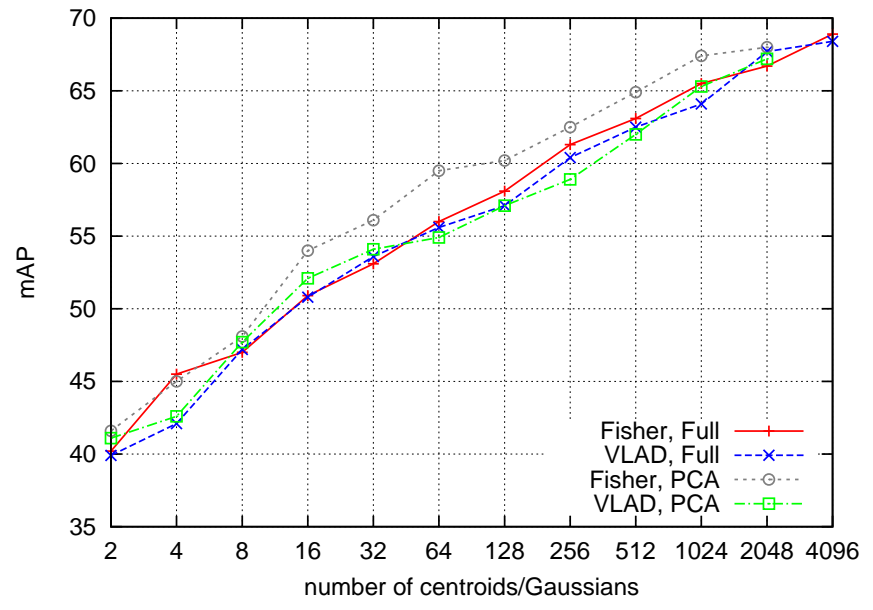

Fig. 2. Comparison of the Fisher and VLAD representations with and without reduction of the local descriptors with PCA (mAP on Holidays).

dataset as a function of $K$. The respective results are similar if these representations are learned and computed on the plain SIFT descriptors, as observed in [23]. However, applying PCA to local descriptors (reduced to 64 dimensions) consistently improves the results of the FV, see Section III-D for possible reasons. This dimensionality reduction does not improve the results for VLAD, probably because it does not benefit from the decorrelating effect of the PCA. As a result, FV+PCA outperforms VLAD by a few points of mAP.

In the rest of the paper, the local descriptors are reduced to 64 dimensions using PCA in all experiments.

Impact of the codebook size. Figure 2 clearly shows that the larger the number of centroids, the better the performance. For $K=4096$ we obtain a $\mathrm{mAP}=68.9 \%$, which outperforms any result reported for standard BOW on this dataset ([35] reports $\mathrm{mAP}=57.2 \%$ with a $200 \mathrm{k}$ vocabulary using the same descriptors). Interestingly, the slope of the curve does not exhibit a saturation for the values of $K$ considered. However, for high values of $K$ the dimensionality of the vector becomes very large and is not compatible with the dimensionality reduction strategy proposed in this paper.

Comparison of BOW/VLAD/FV. The results are presented in Tables I and II. Overall, one can observe that Fisher and VLAD provide competitive results. On Holidays and UKB, both Fisher and VLAD with only $K=64$ outperform the BOW representation. However, BOW is comparatively better when the variability of the objects/images is limited, as for the Oxford Building dataset and the Copydays dataset. In this case, very large vocabularies are necessary and Philbin et al. [1] used up to 1 million visual words, thereby obtaining a $\mathrm{mAP}=49.3 \%$ on Oxford5K when learning the $1 \mathrm{M}$ vocabulary on the Paris dataset ${ }^{4}$ and using soft quantization. FV outperforms these results by increasing $K$ : using the setup of [1] (same descriptors and same learning set), we obtained the following $\mathrm{mAP}$ values:

\footnotetext{
${ }^{4}$ Results obtained when learning the vocabulary on the Oxford Building set itself do not correspond to a realistic setup, as in that case the vocabulary is very specific and not likely to be re-used for other types of images or larger datasets.
}

$\begin{array}{ccccc}K= & 512 & 1024 & 2048 & 4096 \\ \operatorname{mAP}(\%) & 49.2 & 52.8 & 54.5 & 55.3\end{array}$

Dimensionality reduction on BOW/VLAD/FV. Our objective is to obtain a very compact representation. We, therefore, evaluate the performance of each representation when reduced to $D^{\prime}=128$ dimensions. Tables I and II show that the conclusions for the plain representations remain valid. The main difference appears for the Oxford dataset where the PCA has a strong negative impact on the performance, especially in the case of BOW.

Dimension reduction does not necessarily reduce the accuracy, as already observed in [12]. Indeed, Table I shows that a limited reduction tends to improve the accuracy for BOW, Fisher and VLAD. It is also worth noticing that higher dimensional representations, which usually provide better accuracy, suffer more from the dimensionality reduction. This is especially true for Fisher kernels and VLAD: for $D^{\prime}=64$, 128,512 , using only 64 centroids/mixtures is better than using larger values of $K$. Using only $D^{\prime}=128$ dimensions, i.e., the dimensionality of a single SIFT descriptor, Fisher attains the excellent accuracy of $\mathrm{mAP}=56.5 \%$ on Holidays, which is comparable to the result obtained with BOW when using a vocabulary of $200 \mathrm{k}$ visual words.

Comparison with GIST. The global GIST descriptor is very popular in some recognition systems [11]. It was compared with BOW in [19], where it was shown to provide lower results than BOW for image search. However, its performance is competitive in a near duplicate detection context. Table II confirms these observations. It appears that the full 960dimensional GIST descriptor is significantly outperformed by all other representations except for the JPEG5 transformation, which does not change the image geometry. This is true even when BOW/Fisher/VLAD are reduced to a small number of dimensions. Overall, this global descriptor results in a poor performance for image search.

Conclusion. In this section, we have evaluated the performance of VLAD and FV and measured the impact of the different dimensionality reduction steps. In particular, our study explains most of the difference between the results reported for FV in [23] and [22]: the better performance of $\mathrm{FV}$ in [22] is mainly due to the power normalization, which consistently improves the results, and to the dimensionality reduction of the local descriptors by PCA, which is highly beneficial for FV. In this setup, the results of FV are better than those of VLAD on most databases, see Table II. In the following, we adopt the choices that are, on average, the best:

- SIFT descriptors reduced to 64 dimensions by PCA;

- FV with power normalization of the components $(\alpha=$ 0.5 ) followed by a L2 normalization.

\section{FROM VeCtors to CODES}

This section addresses the general problem of coding an image descriptor. Given a $D$-dimensional input vector, we want to produce a code of $B$ bits encoding the image representation, such that the nearest neighbors of a (non-encoded) query vector can be efficiently searched in a set of $n$ encoded database vectors. 


\begin{tabular}{|lrc|cccccc|}
\hline Descriptor & \multicolumn{1}{c|}{$K$} & $D$ & \multicolumn{5}{c|}{ Holidays (mAP) } \\
& & & $D^{\prime}=D$ & $\rightarrow D^{\prime}=2048$ & $\rightarrow D^{\prime}=512$ & $\rightarrow D^{\prime}=128$ & $\rightarrow D^{\prime}=64$ & $\rightarrow D^{\prime}=32$ \\
\hline BOW & 1000 & 1000 & 40.1 & & 43.5 & 44.4 & 43.4 & 40.8 \\
& 20000 & 20000 & 43.7 & 41.8 & 44.9 & 45.2 & 44.4 & 41.8 \\
\hline Fisher $(\mu)$ & 16 & 1024 & 54.0 & & 54.6 & 52.3 & 49.9 & 46.6 \\
& 64 & 4096 & 59.5 & 60.7 & 61.0 & 56.5 & 52.0 & 48.0 \\
& 256 & 16384 & 62.5 & 62.6 & 57.0 & 53.8 & 50.6 & 48.6 \\
\hline VLAD & 16 & 1024 & 52.0 & & 52.7 & 52.6 & 50.5 & 47.7 \\
& 64 & 4096 & 55.6 & 57.6 & 59.8 & 55.7 & 52.3 & 48.4 \\
& 256 & 16384 & 58.7 & 62.1 & 56.7 & 54.2 & 51.3 & 48.1 \\
\hline
\end{tabular}

TABLE I

COMPARISON OF BOW, FISHER AND VLAD REPRESENTATIONS, BEFORE AND AFTER DIMENSION REDUCTION: THE PERFORMANCE IS GIVEN FOR THE FULL $D$-DIMENSIONAL DESCRIPTOR AND AFTER A REDUCTION TO $D^{\prime}$ COMPONENTS WITH PCA. THE NUMBER $K$ STANDS FOR THE NUMBER OF CENTROIDS FOR BOW AND VLAD, AND FOR THE NUMBER OF GAUSSIANS FOR FISHER.

\begin{tabular}{|l|r|c|rr|rr|rr|}
\hline Descriptor & GIST & BOW 200k & BOW 20k & Fisher 64 & \multicolumn{2}{|c|}{ VLAD 64 } \\
PCA to $D^{\prime}=$ & & & & 128 & & 128 & 128 \\
\hline UKB $(4 \times R @ 4)$ & 1.62 & 2.81 & 2.87 & 2.95 & 3.35 & 3.33 & 3.28 & 3.35 \\
Holidays & 36.5 & 54.0 & 43.7 & 45.2 & 59.5 & 56.5 & 55.6 & 55.7 \\
Oxford & & 36.4 & 31.9 & 15.9 & 31.7 & 24.3 & 30.4 & 25.7 \\
Oxford (learned on Paris) & & & 35.4 & 19.4 & 41.8 & 30.1 & 37.8 & 28.7 \\
Copydays JPEG 5 (+10k images) & 100.0 & 90.6 & 100.0 & 100.0 & 93.7 & 63.7 & 94.1 & 72.5 \\
Copydays Crop 50\% (+10k images) & 67.8 & 97.3 & 100.0 & 100.0 & 98.7 & 92.7 & 97.7 & 94.2 \\
Copydays Strong (+10k images) & 27.7 & 70.7 & 54.3 & 26.9 & 59.6 & 41.2 & 59.2 & 42.7 \\
\hline
\end{tabular}

TABLE II

PERFORMANCE OF THE RAW DESCRIPTORS AS WELL AS DESCRIPTORS COMPRESSED TO $D^{\prime}=128$ ON SEVERAL DATASETS, MEASURED BY MAP FOR ALL DATASETS EXCEPT FOR UKB.

We handle this problem in two steps, that must be optimized jointly: 1) a projection that reduces the dimensionality of the vector (see previous section) and 2) a quantization used to index the resulting vectors. We consider the recent approximate nearest neighbor search method of [24], which is briefly described in the next subsection. We will show the importance of the joint optimization by measuring the mean squared Euclidean error generated by each step.

\section{A. Approximate nearest neighbor}

Approximate nearest neighbors search methods [36], [17], [37], [11], [18] are required to handle large databases in computer vision applications [38]. One of the most popular techniques is Euclidean Locality-Sensitive Hashing [36], which has been extended in [17] to arbitrary metrics. However, these approaches and the one of [37] are memory consuming, as multiple hash tables or trees are required. The method of [18], which embeds the vector into a binary space, better satisfies the memory constraint. It is, however, significantly outperformed in terms of the trade-off between memory and accuracy by the product quantization-based approximate search method of [24]. In the following, we use this method, as it offers better accuracy and because the search algorithm provides an explicit approximation of the indexed vectors. This allows us to compare the vector approximations introduced by the dimensionality reduction and the quantization, respectively. We use the asymmetric distance computation (ADC) variant of this approach, which only encodes the vectors of the database, but not the query vector. This method is summarized in the following.

a) ADC approach.: Let $x \in \mathbb{R}^{D}$ be a query vector and $\mathcal{Y}=\left\{y_{1}, \ldots, y_{n}\right\}$ a set of vectors in which we want to find the nearest neighbor $\mathrm{NN}(x)$ of $x$. The ADC approach consists in encoding each vector $y_{i}$ by a quantized version $c_{i}=q\left(y_{i}\right) \in \mathbb{R}^{D}$. For a quantizer $q($.$) with k$ centroids, the vector is encoded by $B=\log _{2}(k)$ bits, $k$ being a power of 2 . Finding the $a$ nearest neighbors $\mathrm{NN}_{a}(x)$ of $x$ simply consists in computing

$$
\mathrm{NN}_{a}(x)=a-\arg \min _{i}\left\|x-q\left(y_{i}\right)\right\|^{2} .
$$

Note that, in contrast with the embedding method of [18], the query $x$ is not converted to a code: there is no approximation error on the query side.

To get a good vector approximation, $k$ should be large $(k=$ $2^{64}$ for a 64 bit code). For such large values of $k$, learning a $k$-means codebook as well as assigning to the centroids is not tractable. Our solution is to use a product quantization method which defines the quantizer without explicitly enumerating its centroids. A vector $x$ is first split into $m$ subvectors $x^{1}, \ldots x^{m}$ of equal length $D / m$. A product quantizer is then defined as a function

$$
q(x)=\left(q_{1}\left(x^{1}\right), \ldots, q_{m}\left(x^{m}\right)\right),
$$

which maps the input vector $x$ to a tuple of indices by separately quantizing the subvectors. Each individual quantizer 
$q_{j}($.$) has k_{\mathrm{s}}$ reproduction values learned by k-means. To limit the assignment complexity $\mathcal{O}\left(m \times k_{\mathrm{S}}\right), k_{\mathrm{S}}$ is a small value (e.g., $k_{\mathrm{s}}=256$ ). However, the set $k$ of centroids induced by the product quantizer $q($.$) is large: k=\left(k_{\mathrm{s}}\right)^{m}$.

The square distances in Equation 21 are computed using the decomposition

$$
\left\|x-q\left(y_{i}\right)\right\|^{2}=\sum_{j=1, \ldots, m}\left\|x^{j}-q_{j}\left(y_{i}^{j}\right)\right\|^{2},
$$

where $y_{i}^{j}$ is the $j^{\text {th }}$ subvector of $y_{i}$. The square distances in this summation are read from look-up tables computed, prior to the search, between each subvector $x^{j}$ and the $k_{\mathrm{s}}$ centroids associated with the corresponding quantizer $q_{j}$. The generation of the tables is of complexity $\mathcal{O}\left(D \times k_{\mathrm{s}}\right)$. When $k_{\mathrm{s}} \ll n$, this complexity is negligible compared with the summation cost of $\mathcal{O}(D \times n)$ in Equation 21 .

This quantization method offers an explicit vector approximation: a database vector $y_{i}$ can be decomposed as

$$
y_{i}=q\left(y_{i}\right)+\varepsilon_{\mathrm{q}}\left(y_{i}\right),
$$

where $q\left(y_{i}\right)$ is the centroid associated with $y_{i}$ and $\varepsilon_{\mathrm{q}}\left(y_{i}\right)$ the error vector generated by the quantizer.

Notation: ADC $m \times b_{\mathrm{s}}$ refers to the method when using $m$ subvectors and $b_{\mathrm{s}}$ bits to encode each subvector $\left(b_{\mathrm{s}}=\log _{2} k_{\mathrm{s}}\right)$. The total number of bits $B$ used to encode a vector is then given by $B=m b_{\mathrm{s}}$.

\section{B. Indexation-aware dimensionality reduction}

Dimensionality reduction is an important step in approximate nearest neighbor search, as it impacts the subsequent indexing. In this section, for the ADC approach, we express the tradeoff between this operation and the indexing scheme using a single quality measure: the approximation error. For the sake of presentation, we assume that the mean of each vector component is 0 . By construction, this is approximately the case for Fisher ${ }^{5}$ and VLAD vectors.

The $D^{\prime} \times D$ PCA matrix $M$ maps descriptor $x \in \mathbb{R}^{D}$ to the transformed descriptor $x^{\prime}=M x \in \mathbb{R}^{D^{\prime}}$. It is the upper part of an orthogonal matrix. This dimensionality reduction can also be interpreted in the initial space as a projection. In that case, $x$ is approximated by

$$
x_{\mathrm{p}}=x-\varepsilon_{\mathrm{p}}(x)
$$

where the error vector $\varepsilon_{\mathrm{p}}(x)$ lies in the null space of $M$. The vector $x_{\mathrm{p}}$ is related to $x^{\prime}$ by the pseudo-inverse of $M$, which is the transpose of $M$ in this case. Therefore, the projection is $x_{\mathrm{p}}=M^{\top} M x$. For the purpose of indexing, the vector $x^{\prime}$ is subsequently encoded as $q\left(x^{\prime}\right)$ using the ADC approach, which can also be interpreted in the original $D$-dimensional space as the approximation ${ }^{6}$

$$
q\left(x_{\mathrm{p}}\right)=x-\varepsilon_{\mathrm{p}}(x)-\varepsilon_{\mathrm{q}}\left(x_{\mathrm{p}}\right)
$$

${ }^{5}$ This comes from the property $E_{x \sim u_{\lambda}} \nabla_{\lambda} \log u_{\lambda}(x)=$ $\nabla_{\lambda} E_{x \sim u_{\lambda}} \log u_{\lambda}(x) \approx 0$ if $u_{\lambda}$ is estimated with MLE.

${ }^{6}$ For the sake of conciseness, the quantities $M^{T} q\left(x^{\prime}\right)$ and $M^{T} \varepsilon_{\mathrm{q}}\left(x^{\prime}\right)$ are simplified to $q\left(x_{\mathrm{p}}\right)$ and $\varepsilon_{\mathrm{q}}\left(x_{\mathrm{p}}\right)$ respectively. where $\varepsilon_{\mathrm{p}}(x) \in \operatorname{Null}(M)$ and $\varepsilon_{\mathrm{q}}\left(x_{\mathrm{p}}\right) \in \operatorname{Null}(M)^{\perp}$ (because the ADC quantizer is learned in the principal subspace) are orthogonal. At this point, we make two observations:

1) Due to the PCA, the variance of the different components of $x^{\prime}$ is not balanced. Therefore the ADC structure, which allocates a fixed number of bits per subvector, quantizes the first principal components more coarsely in comparison with the last components, leading to a high quantization error on the first components. In order to address this problem, it is possible to balance the components' variance by applying an orthogonal transformation after the PCA. In [23], two strategies are compared. First a Householder matrix is learned to perfectly balance the energy on the components. The second strategy simply consists in applying a random rotation matrix after the PCA. Both approaches improve the results, and the random matrix is shown to be as effective as the learned one. We therefore adopt this choice in the following.

2) There is a trade-off on the number of dimensions $D^{\prime}$ to be retained by the PCA. If $D^{\prime}$ is large, the projection error vector $\varepsilon_{\mathrm{p}}(x)$ is of limited magnitude, but a large quantization error $\varepsilon_{\mathrm{q}}\left(x_{\mathrm{p}}\right)$ is introduced. On the other hand, keeping a small number of components leads to a high projection error and a low quantization error.

Joint optimization of reduction/indexing. Let us now consider the second problem, i.e., optimizing the dimension $D^{\prime}$, having fixed a constraint on the number of bits $B$ used to represent the $D$-dimensional vector $x$, for instance $B=128$ (16 bytes). The squared Euclidean distance between the reproduction value and $x$ is the sum of the errors $\left\|\varepsilon_{\mathrm{p}}(x)\right\|^{2}$ and $\left\|\varepsilon_{\mathrm{q}}\left(x_{\mathrm{p}}\right)\right\|^{2}$, both of which depend on the selected $D^{\prime}$. The mean square error $e\left(D^{\prime}\right)$ is empirically measured on a learning vector set $\mathcal{L}$ as

$$
\begin{aligned}
e\left(D^{\prime}\right) & =e_{p}\left(D^{\prime}\right)+e_{q}\left(D^{\prime}\right) \\
& =\frac{1}{\operatorname{card}(\mathcal{L})} \sum_{x \in \mathcal{L}}\left\|\varepsilon_{\mathrm{p}}(x)\right\|^{2}+\left\|\varepsilon_{\mathrm{q}}\left(x_{\mathrm{p}}\right)\right\|^{2} .
\end{aligned}
$$

This gives us an objective criterion to optimize directly the dimensionality, which is obtained by finding on the learning set the value of $D^{\prime}$ minimizing this criterion.

\section{Remarks.}

- The selection of $D^{\prime}$ using this mean square error minimization is not optimized with respect to an image search criterion. Note however minimizing this error appears to be a reasonable choice, as empirically observed in [23].

- In the ADC indexing scheme, $D^{\prime}$ must be a multiple of $m$. For instance, by keeping $D^{\prime}=64$ eigenvalues, the valid set of values for $m$ is $\{1,2,4,8,16,32,64\}$.

The impact of dimensionality reduction and indexation based on ADC is illustrated by the VLAD pictorial representation introduced in Section III. We can present the projected and quantized VLAD in this form, as both the PCA projection and the quantization provide a way of reconstructing the projected/quantized vector. Figure 3 illustrates how each 


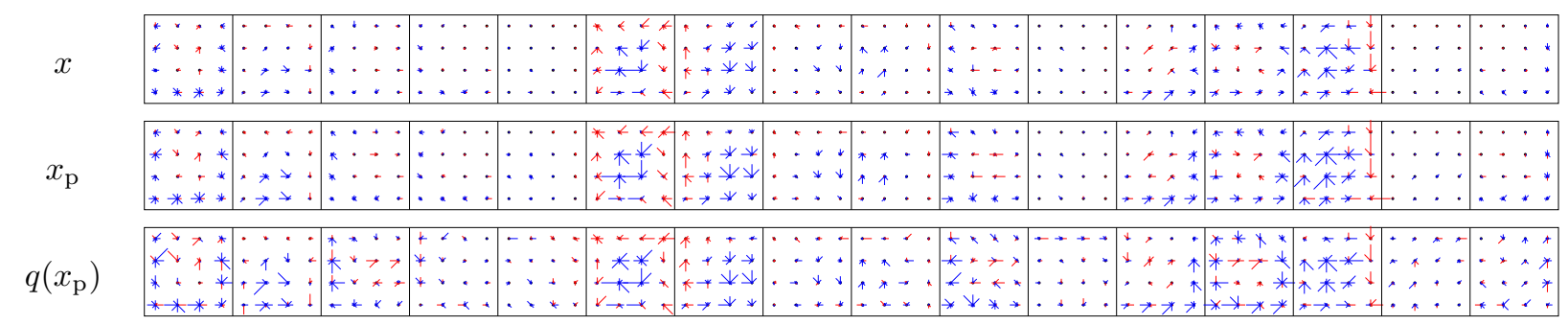

Fig. 3. Effect of the encoding steps on the descriptor. Top: VLAD vector $x$ for $K=16(D=2048)$. Middle: vector $x_{\mathrm{p}}$ altered by the projection onto the PCA subspace $\left(D^{\prime}=128\right)$. Bottom: vector $q\left(x_{\mathrm{p}}\right)$ after indexing by ADC $16 \times 8$ (16-bytes code).

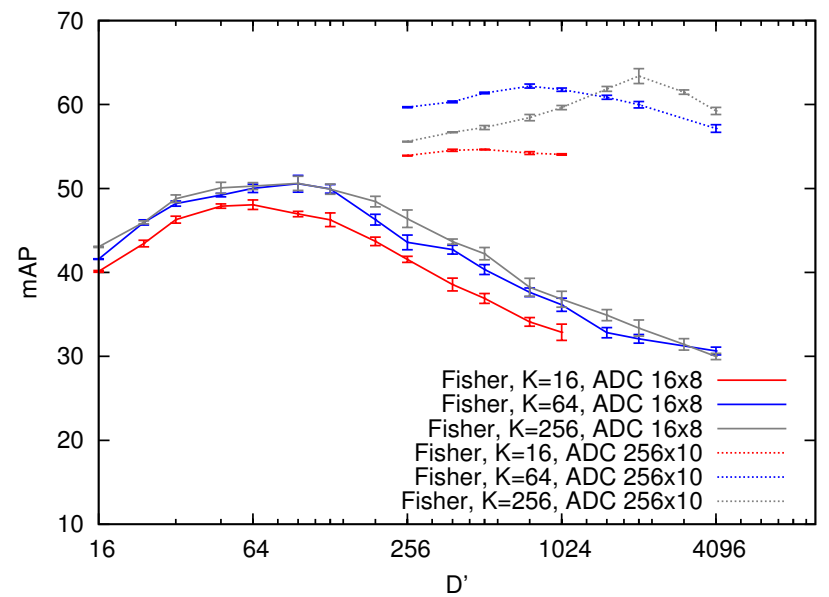

Fig. 4. Search on Holidays with ADC $16 \times 8$ and ADC $256 \times 10$ representations, for reduction to varying dimensions $D^{\prime}$. Experiments are averaged over 5 learning runs (error bars $=$ standard deviations over the runs).

of these operations impacts our representation. One can see that the vector is only slightly altered, even for a compact representation of $B=16$ bytes.

\section{EXPERIMENTS}

In this section, we evaluate the performance of the Fisher vector when used with the joint dimensionality reduction/indexing approach of Section V. This evaluation uses the improved FV representation of [22] in conjunction with the indexing scheme of [23]. Our comparison focuses on the UKB and Holidays datasets. Large scale experiments on Holidays+Flickr10M were used to measure search accuracy and efficiency on a large scale of 10 million images. As we focus on the intrinsic quality of the large scale system, we do not apply the re-ranking stage which is usually performed on a shortlist to filter out geometrically inconsistent results [1], [35].

\section{A. Dimensionality reduction and indexation}

Given an image representation with a vector of length $D$ and a fixed number $B$ of bits to encode this vector, Figure 4 confirms the analysis of Section V: there is an important variation with respect to $D^{\prime}$. A method to fix $D^{\prime}$ consists in minimizing the total square error introduced by the projection and the quantization steps, as suggested in V-B. This choice may not be optimal with respect to image search quality measured by, e.g., mAP. However, as the performance is stable

\begin{tabular}{|lrrr|}
\hline Method & bytes & UKB & Holidays \\
\hline BOW, $K=20,000$ & 10364 & 2.87 & 43.7 \\
BOW, $K=200,000$ & 12886 & 2.81 & 54.0 \\
\hline miniBOF [12] & 20 & 2.07 & 25.5 \\
& 80 & 2.72 & 40.3 \\
& 160 & 2.83 & 42.6 \\
\hline FV $K=64$, spectral hashing 128 bits & 16 & 2.57 & 39.4 \\
\hline VLAD, $K=16$, ADC 16×8 [23] & 16 & 2.88 & 46.0 \\
VLAD, $K=64$, ADC 32 $\times 10[23]$ & 40 & 3.10 & 49.5 \\
\hline FV $K=8$, binarized [22] & 65 & 2.79 & 46.0 \\
FV $K=64$, binarized $[22]$ & 520 & 3.21 & 57.4 \\
\hline FV $K=64$, ADC $16 \times 8\left(D^{\prime}=96\right)$ & $\mathbf{1 6}$ & $\mathbf{3 . 1 0}$ & $\mathbf{5 0 . 6}$ \\
FV $K=256$, ADC $256 \times 10\left(D^{\prime}=2048\right)$ & $\mathbf{3 2 0}$ & $\mathbf{3 . 4 7}$ & $\mathbf{6 3 . 4}$ \\
\hline
\end{tabular}

TABLE III

COMPARISON OF OUR APPROACH WITH THE STATE OF THE ART ON UKB (SCORE/4) AND HOLIDAYS (MAP).

around the optimal value of $D^{\prime}$ (see Figure 4), in practice the proposed optimization procedure provides close-to-optimal results.

\section{B. Comparison with the state of the art}

Table III and Figure 5 compare the performance obtained by our indexing scheme to the state of the art on the Holidays and UKB datasets. Compared to the miniBOF approach of [12], the proposed approach is significantly more precise at all operating points. Compared to BOW, our approach obtains a comparable search quality with about two orders of magnitude less memory. With respect to the approaches [22] and [23], a significant improvement is obtained by using the improved FV of [22] jointly with the indexing method of [23].

Figure 5 also illustrates the trade-off between search quality and memory usage. Interestingly, the best choice for the number $K$ of Gaussians depends on the number of bits $B$ chosen to represent the image. Compared to BOW, which gives $\mathrm{mAP}=54 \%$ for a $200 \mathrm{k}$ vocabulary, a competitive accuracy of $\mathrm{mAP}=55.2 \%$ is obtained with only 32 bytes. Note that small (resp. large) values of $K$ should be associated with small (resp. large) values of $B$, as they are more impacted by dimensionality reduction. On this figure, the variant $K=16$ is never selected as the best, which explains why only $K=64$ and $K=256$ appear in the plot.

Table III also compares our indexing scheme to the spectral hashing [18] coding scheme. For a memory usage of 16 bytes, ADC outperforms spectral hashing by more than 10 points of mAP on Holidays. Similarly, it is possible to use significantly 


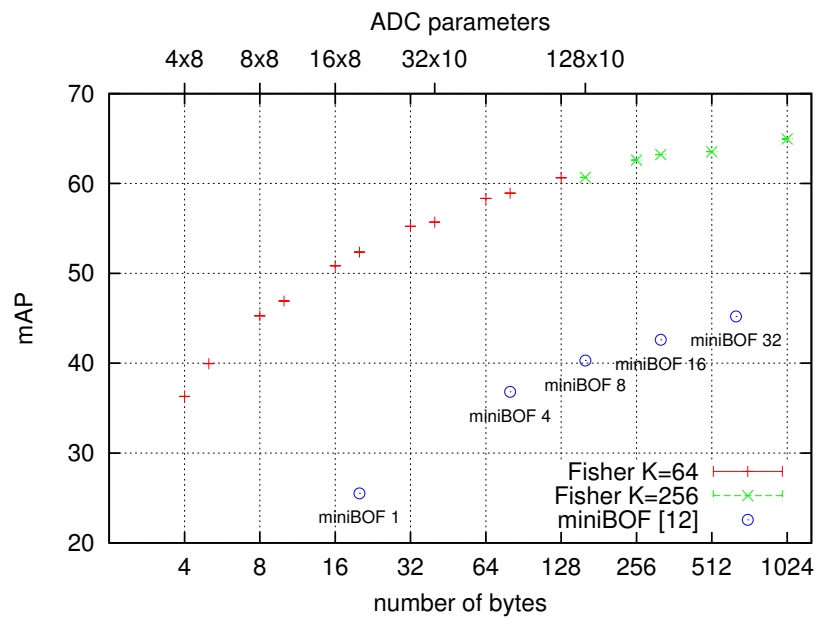

Fig. 5. mAP for a search on Holidays with varying ADC quantization parameters (number of bytes). Results of [12] are reported with circles for reference.

less memory to attain the same performance: using 5 bytes per image in $\mathrm{ADC}$ achieves the same $\mathrm{mAP}(\approx 40 \%)$ as $\mathrm{SH}$ with 16 bytes, see Figure 5.

\section{Large-scale experiments}

1) Experiments on Holidays and Flickr10M: Figure 6 shows the behavior of our approach on a large scale. We have constructed a dataset by combining the images from Holidays with a subset of Flickr10M of increasing size. Recall that, in such a large dataset, the number of outliers is very important. The challenge is to rank the relevant images well enough to ensure that geometrical verification will validate them in a re-ranking stage, which can typically be performed for a few hundred images only.

For this experiment, we have used the non exhaustive search variant of ADC, called IVFADC. IVFADC combines ADC with an inverted file to restrict the search to a subset of vectors: in our case, only 64 lists of coded descriptors are visited out of 8192 lists in total. Consequently, it stores the image identifiers explicitly, which requires an extra 4 bytes of memory per image (see [24] for details). Compared to ADC, on large datasets IVFADC is one or two orders of magnitude faster and gives slightly better results.

The mAP performance is displayed as a function of the dataset size. We report the results for

- the plain Fisher vector ( $K=64, D=4096)$;

- the same Fisher vector reduced to $D^{\prime}=96$ dimensions by PCA;

- these PCA-reduced FV indexed by IVFADC with $16 \times 8$ codes, i.e., $16+4=20$ bytes per indexed image. We also present a more expensive operating point, for which $K=256, D^{\prime}=2048$, and $256 \times 10$ codes have been used, leading to a representation of 324 bytes in memory.

The results are significantly better than those reported in [12], where a mAP of $6.6 \%$ is reported for 1 million images and a 20-bytes representation, against $\mathrm{mAP}=27.9 \%$ for the same memory usage with the proposed method, and $\mathrm{mAP}=37.0 \%$ when using 324 bytes. Note that this is also an

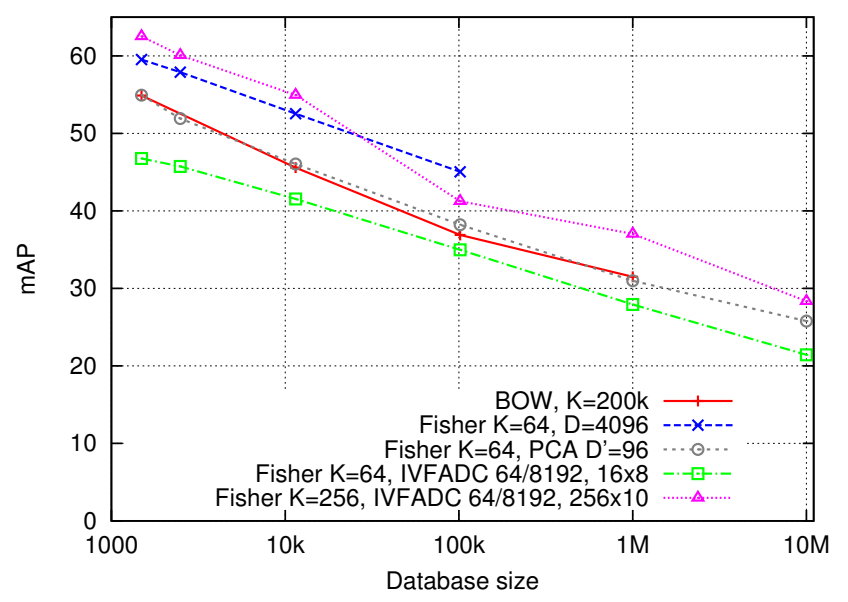

Fig. 6. Search accuracy as a function of the database size.

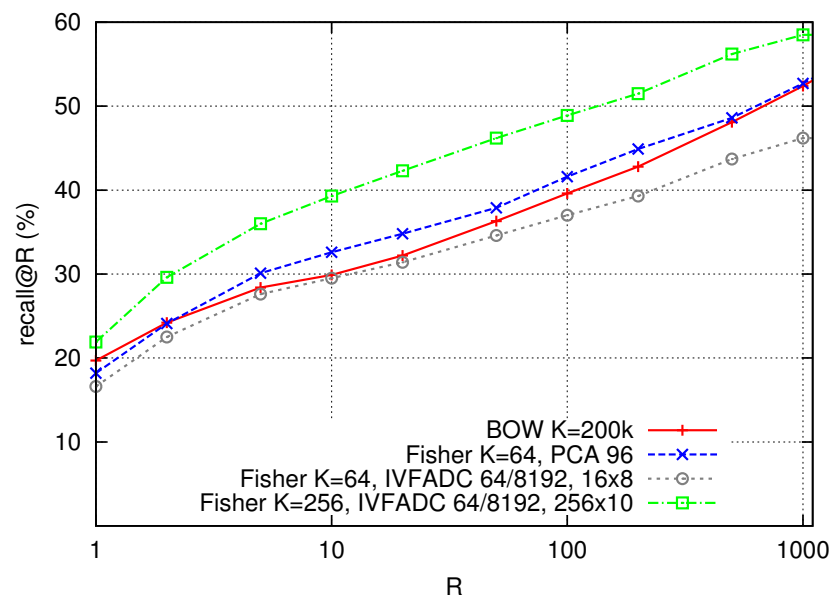

Fig. 7. Quality of the shortlist (of varying size $R$ ): recall@ $R$ when searching in 1 million images.

improvement over [23], where we obtained $\mathrm{mAP}=24.1 \%$ using the VLAD vector. Interestingly, the 96-dimensional vector obtained by PCA offers results comparable to those of BOW with a large vocabulary.

In order to measure how our system would benefit from being used in conjunction with a post-verification scheme, Figure 7 gives the recall $@ R$ as a function of the number $R$. It can be interpreted as the rate of relevant images that will be geometrically verified if we consider that a verification is applied on a short-list of $R$ images (typically, $R$ is limited to 100). The experiment is limited to Holidays+Flickr1M because BOW does not scale to 10 million images. With a representation of 20 bytes, the proposed approach is almost as accurate as BOW, and becomes significantly better when increasing the size of the codes.

2) Experiments on Copydays and Exalead100M: Given the compactness of our image descriptor encoding, our method can scale up to one billion images with a reasonable amount of RAM (for instance, 20GB when using only 20 bytes per image). Only a few systems are able to work on such a scale, an example is the GISTIS approach of [19]. This approach 


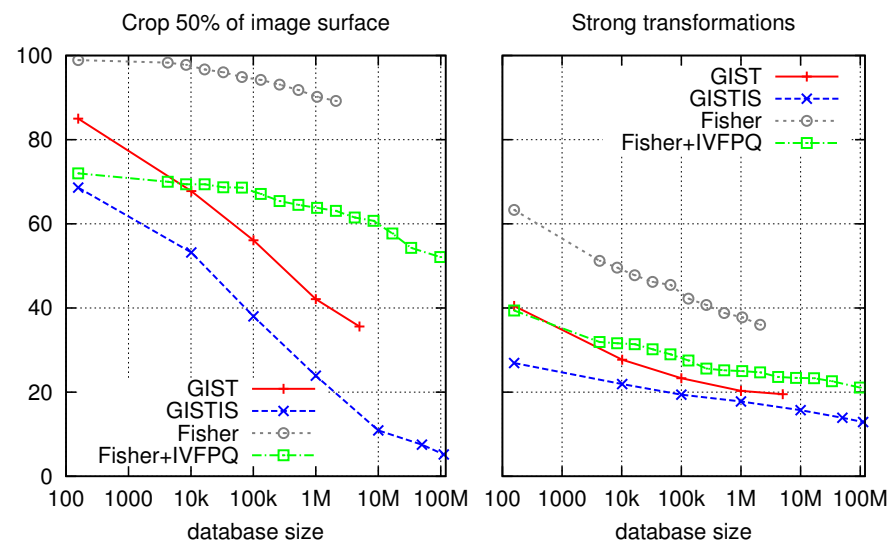

Fig. 8. Copydays retrieval results (mAP), for two types of transformations and a varying number of distractors.

combines the GIST descriptor [16] with an indexing method derived from [26]. Results are reported on 110 million tiny images $(32 \times 32$ pixels $)$. In the following, we report a comparison of our approach with the GISTIS method for Copydays merged with the Exalead100M dataset (See Section II for a description). Since the images are smaller in this setup (150 pixels in their larger dimension), the local descriptor's threshold (on cornerness) is reduced to increase the number of descriptors input to the Fisher computation.

Figure 8 shows the results for two subsets of Copydays: crop 50\% (157 transformed queries, 1 per database image) and strong transformations (229 query images, each of which has only 1 matching image in the database). The number of distracting images from Exalead100M is increased up to the full dataset (100M images). We can observe that the uncompressed Fisher descriptor $(K=64, D=4096)$ is clearly more discriminant than the uncompressed color GIST descriptor ( $D=960$ ). The IVFADC configuration (IVF 64/8192, $64 \times 8$ ) was selected to allocate the same size per descriptor as the GISTIS indexing method: 68 bytes per image. The results after indexing are better than for GISTIS. Moreover, the slope of the curves shows that our approach is less sensitive to distractors: the comparative performance is much better for our scheme as the database grows.

Timings: All timing experiments have been performed on a single processor core. On average, searching our 100 million dataset with the IVFADC indexing structure $(64 \times 8$ codes, 64 lists visited out of 8192) takes $245 \mathrm{~ms}$. This efficiency is at least two orders of magnitude above the BOW: [35] reports a query time of $620 \mathrm{~ms}$ on a quad-core processor to search in 1 million images given a vocabulary of $K=200 \mathrm{k}$ visual words. The time search is $50 \%$ higher than the one of GISTIS (160ms), but for a significantly better search quality.

\section{CONCLUSION}

Many state-of-the-art large-scale image search systems follow the same paradigm: statistics computed from local invariant features are aggregated into an image-level vector signature which is subsequently compressed and indexed for computational and memory efficiency. The BOW histogram has become a standard for the aggregation part. For the compression part, most approaches use binary embeddings. This article departs from this paradigm in two ways.

We first propose to use the Fisher kernel framework for the local feature aggregation. This representation is shown to yield high performance and its accuracy remains competitive even after a significant dimensionality reduction to 128 dimensions, i.e., of the same size as a single SIFT vector.

Secondly, we employ an asymmetric product quantization scheme for the vector compression part, and jointly optimize the dimensionality reduction and compression. Impressive search results are achieved with a tiny binary code, e.g., a mAP of $50.6 \%$ on Holidays with 128 bits signatures. With such a small memory footprint, one billion images fit in the RAM of a 20GB server. We achieve a response time of $250 \mathrm{~ms}$ on a 100 million image dataset on a single processor core.

\section{ACKNOWLEDGEMENTS}

We thank the company Exalead for providing the corpus of 100M images. This work was partially funded by the European project PINVIEW, by the QUAERO project (supported by OSEO, French State agency for innovation), by the European integrated project AXES, and by the ANR project GAIA.

\section{REFERENCES}

[1] J. Philbin, O. Chum, M. Isard, J. Sivic, and A. Zisserman, "Object retrieval with large vocabularies and fast spatial matching," in CVPR, June 2007.

[2] D. Nistér and H. Stewénius, "Scalable recognition with a vocabulary tree," in CVPR, pp. 2161-2168, June 2006.

[3] Z. Wu, Q. Ke, M. Isard, and J. Sun, "Bundling features for large scale partial-duplicate web image search," in CVPR, pp. 25-32, 2009.

[4] J. Law-To, L. Chen, A. Joly, I. Laptev, O. Buisson, V. Gouet-Brunet, N. Boujemaa, and F. Stentiford, "Video copy detection: a comparative study," in CIVR, (New York, NY, USA), pp. 371-378, ACM, 2007.

[5] M. Everingham, L. Van Gool, C. K. I. Williams, J. Winn, and A. Zisserman, "The PASCAL visual object classes (VOC) challenge," International Journal of Computer Vision, vol. 88, pp. 303-338, June 2010.

[6] J. Sivic and A. Zisserman, "Video Google: A text retrieval approach to object matching in videos," in ICCV, pp. 1470-1477, October 2003.

[7] D. Lowe, "Distinctive image features from scale-invariant keypoints," International Journal of Computer Vision, vol. 60, no. 2, pp. 91-110, 2004.

[8] K. Mikolajczyk and C. Schmid, "A performance evaluation of local descriptors," IEEE Transactions on Pattern Analysis and Machine Intelligence, vol. 27, no. 10, pp. 1615-1630, 2005.

[9] S. Winder and M. Brown, "Learning local image descriptors," in CVPR, June 2007.

[10] S. Winder, G. Hua, and M. Brown, "Picking the best Daisy," in CVPR, June 2009.

[11] A. Torralba, R. Fergus, and Y. Weiss, "Small codes and large databases for recognition," in CVPR, June 2008.

[12] H. Jégou, M. Douze, and C. Schmid, "Packing bag-of-features," in ICCV, September 2009.

[13] O. Chum, M. Perdoch, and J. Matas, "Geometric min-hashing: Finding a (thick) needle in a haystack," in CVPR, June 2009.

[14] O. Chum, J. Philbin, and A. Zisserman, "Near duplicate image detection: min-hash and tf-idf weighting," in $B M V C$, September 2008.

[15] L. Torresani, M. Szummer, and A. Fitzgibbon, "Learning querydependent prefilters for scalable image retrieval," in CVPR, June 2009.

[16] A. Oliva and A. Torralba, "Modeling the shape of the scene: a holistic representation of the spatial envelope," International Journal of Computer Vision, vol. 42, no. 3, pp. 145-175, 2001.

[17] B. Kulis and K. Grauman, "Kernelized locality-sensitive hashing for scalable image search," in ICCV, October 2009.

[18] Y. Weiss, A. Torralba, and R. Fergus, "Spectral hashing," in NIPS, 2008. 
[19] M. Douze, H. Jégou, H. Singh, L. Amsaleg, and C. Schmid, "Evaluation of GIST descriptors for web-scale image search," in CIVR, July 2009.

[20] T. Jaakkola and D. Haussler, "Exploiting generative models in discriminative classifiers," in NIPS, 1998.

[21] F. Perronnin and C. R. Dance, "Fisher kernels on visual vocabularies for image categorization," in CVPR, June 2007.

[22] F. Perronnin, Y. Liu, J. Sanchez, and H. Poirier, "Large-scale image retrieval with compressed Fisher vectors," in CVPR, June 2010.

[23] H. Jégou, M. Douze, C. Schmid, and P. Pérez, "Aggregating local descriptors into a compact image representation," in CVPR, June 2010.

[24] H. Jégou, M. Douze, and C. Schmid, "Product quantization for nearest neighbor search," IEEE Transactions on Pattern Analysis \& Machine Intelligence, vol. 33, pp. 117-128, January 2011.

[25] K. Mikolajczyk, T. Tuytelaars, C. Schmid, A. Zisserman, J. Matas, F. Schaffalitzky, T. Kadir, and L. V. Gool, "A comparison of affine region detectors," International Journal of Computer Vision, vol. 65, no. 1/2, pp. 43-72, 2005.

[26] H. Jégou, M. Douze, and C. Schmid, "Hamming embedding and weak geometric consistency for large scale image search," in $E C C V$, October 2008.

[27] J. Philbin, O. Chum, M. Isard, J. Sivic, and A. Zisserman, "Lost in quantization: Improving particular object retrieval in large scale image databases," in CVPR, June 2008.

[28] J. van Gemert, C. Veenman, A. Smeulders, and J. Geusebroek, "Visual word ambiguity," IEEE Transactions on Pattern Analysis and Machine Intelligence, vol. 32, pp. 1271-1283, July 2010.

[29] H. Jégou, M. Douze, and C. Schmid, "On the burstiness of visual elements," in CVPR, June 2009.

[30] J. Winn, A. Criminisi, and T. Minka, "Object categorization by learned universal visual dictionary," in ICCV, 2005.

[31] F. Perronnin, J. Sánchez, and Y. Liu, "Large-scale image categorization with explicit data embedding," in CVPR, 2010.

[32] A. Vedaldi and A. Zisserman, "Efficient additive kernels via explicit feature maps," in CVPR, 2010.

[33] X. Zhang, Z. Li, L. Zhang, W. Ma, and H.-Y. Shum, "Efficient indexing for large-scale visual search," in ICCV, October 2009.

[34] C. M. Bishop, Pattern Recognition and Machine Learning. Springer, 2007.

[35] H. Jégou, M. Douze, and C. Schmid, "Improving bag-of-features for large scale image search," International Journal of Computer Vision, vol. 87, pp. 316-336, February 2010.

[36] M. Datar, N. Immorlica, P. Indyk, and V. Mirrokni, "Locality-sensitive hashing scheme based on p-stable distributions," in Proceedings of the Symposium on Computational Geometry, pp. 253-262, 2004.

[37] M. Muja and D. G. Lowe, "Fast approximate nearest neighbors with automatic algorithm configuration," in VISAPP, February 2009.

[38] G. Shakhnarovich, T. Darrell, and P. Indyk, Nearest-Neighbor Methods in Learning and Vision: Theory and Practice, ch. 3. MIT Press, March 2006. 